\title{
Comparison of Submillinewton Thrust Measurements Between a Laser Interferometer and a Load Cell on a Pendulum Balance
}

\author{
Dimitrios Tsifakis *, Christine Charles and Rod Boswell \\ Space Plasma, Power and Propulsion Laboratory, Research School of Physics, The Australian National University, Canberra,
} NSW, Australia

Reliable measurements of thrust from systems to be flown on satellites are essential to

OPEN ACCESS

Edited by:

Robert LeMoyne,

Northern Arizona University,

United States

Reviewed by:

Haibin Tang,

Beihang University, China

Aaron Knoll,

Imperial College London,

United Kingdom

Joe Khachan,

The University of Sydney, Australia Sabrina Pottinger,

Telespazio Spa, Italy Thomas Harle,

Fourth State Medicine Ltd., United Kingdom

*Correspondence:

Dimitrios Tsifakis

Dimitrios.Tsifakis@anu.edu.au

Specialty section:

This article was submitted to Advanced Space Propulsion,

a section of the journal

Frontiers in Space Technologies

Received: 23 November 2020

Accepted: 29 March 2021

Published: 27 April 2021

Citation:

Tsifakis D, Charles $C$ and Boswell $R$ (2021) Comparison of Submillinewton

Thrust Measurements Between a Laser Interferometer and a Load Cell on a Pendulum Balance.

Front. Space Technol. 2:632358.

doi: 10.3389/frspt.2021.632358 ensure repeatable maneuvering capability of small nanosatellites. Thrusters can be used to vary spacecraft orientation, detumbling, and orbit change. Tests have been conducted in a low-pressure vacuum system using a cold gas prototype thruster and two independently calibrated methods: a four-point pendulum with a laser interferometer displacement sensor and a load cell, both of which have measurement capabilities from tens of micronewtons to tens of millinewtons. The agreement is very good, lending confidence in both methods. The advantages and disadvantages of both methods will be discussed. They include absolute accuracy, low thrust accuracy, temporal resolution, simplicity of operation, cost, and sensitivity to vibrations generated by laboratory equipment such as pumps, fans, bumps, and human movement.

Keywords: thrust measurement, propulsion, cubesat, load cell, thruster

\section{INTRODUCTION}

Small satellites having unit dimensions of $10 \mathrm{~cm} \times 10 \mathrm{~cm} \times 10 \mathrm{~cm}$ and mass up to $1.33 \mathrm{~kg}$ (California Polytechnic State University, 2014) or multiples of this have become increasingly popular of late since they can be constructed from simple commercial off-the-shelf (COTS) components and systems. This has allowed small research groups in universities, small to medium enterprises, and hobbyists to engage in a variety of areas of space research and engineering. As of 2020, over 1,300 nanosats and cubesats have already been placed in orbit, but according to the Nanosat database (Kulu, 2019), only about 5\% have propulsion modules and, of these, very little information on the actual testing procedures, laboratory performance, and whether the thruster was successful in flight is publicly available. Examples of publications that demonstrate successful use of a thruster system on nanosatellites include the formation flying of CanX-4 and CanX-5 (Bonin et al., 2015) and, more recently, the NanoFEEP propulsion system on UWE-4 (Kramer et al., 2020). Of primary interest to the further development of nanosatellites is the propulsion module and, in particular, the direct thrust measurement under space conditions. A common technique used for thrust measurement is mounting the thruster on a pendulum and measuring the pendulum displacement when the thruster is activated. Usually, the displacement is measured directly, using a laser interferometer. This paper compares direct thrust measurements of a cold gas thruster using a pendulum thrust balance equipped with a sensitive laser interferometer to using a load cell (force sensor) to measure the thrust directly with the thruster installed on the same pendulum. The combination of a pendulum and a load cell results in a cost-effective system for direct 
thrust measurement compared to the alternative existing methods. This is of interest to entry level cubesat operators for firsthand evaluation of their thrusters.

\subsection{Pendulum Displacement Method}

One of the most typical techniques used for thrust measurement involves the concept of the pendulum. In its simplest form, a pendulum thrust balance can be based on a hanging pendulum (Charles et al., 2016; Wong et al., 2012), an inverted pendulum (Haag, 1991; Kokal and Celik, 2017; Asakawa et al., 2020), or be a torsional balance (Ziemer, 2001; Zhou et al., 2013; Soni and Roy, 2013; Little and Jugroot, 2019; Tang et al., 2011; Zhang et al., 2017). There are also more complicated implementations of this, such as the stand for vertically oriented thrust measurement system described in Moeller and Polzin (2010). In all cases, the application of thrust results in a displacement which is a function of the thrust. The displacement is commonly measured with a laser interferometer or a linear differential voltage transformer (LDVT). The theory of operation of all of these methods is described in detail by Polk et al. (2017). In order to keep the test platform stable and horizontal, it is common to attach rigid arms with metallic flexures at both ends, to each corner of a rectangular plate; the other end of the arms is fixed some distance vertically above. The metallic flexures are oriented such that the movement caused by the action of the thruster is allowed. Together with gravity, the flexures contribute to the restoring force of the pendulum. When the thruster is activated, the distance away from the normal can be determined with a laser interferometer and when correctly calibrated, yields a thrust proportional to displacement. The sensitivity of the system is proportional to the suspended mass, which includes the mass of the thruster under test. An elementary characteristic of a pendulum is that it will oscillate, and given that there is no air friction in vacuum, it may take a very long time for the system to come to rest. These oscillations can be damped actively using solenoids or simply with a magnet generating eddy currents in a metal tube. An alternative to using the displacement of a pendulum for thrust measurement is placing a solenoid and a magnet in a feedback loop which supplies the current required to prevent all motion. In this case, the force of the thruster will be proportional to the current through the solenoid necessary to prevent movement. A benefit of this method is that the natural frequency of the pendulum does not affect the measured values; however, the measurement bandwidth will be limited by the frequency of the feedback loop.

\subsection{Momentum Flux Measurement Method}

An indirect method of measuring thrust consists in measuring the momentum flux of the exhausted material impinging on a target placed near the exhaust of the thruster. The force measured on the target together with modeling of the exhaust plume of the thruster can then be used to infer the thrust produced by the thruster under test. In this method, care needs to be taken to ensure the target does not interfere with the normal operation of the thruster. Typical measurements of momentum thrust have been performed by Chavers and Chang-Díaz (2002), West et al. (2009), and Takahashi et al. (2015), the latter containing

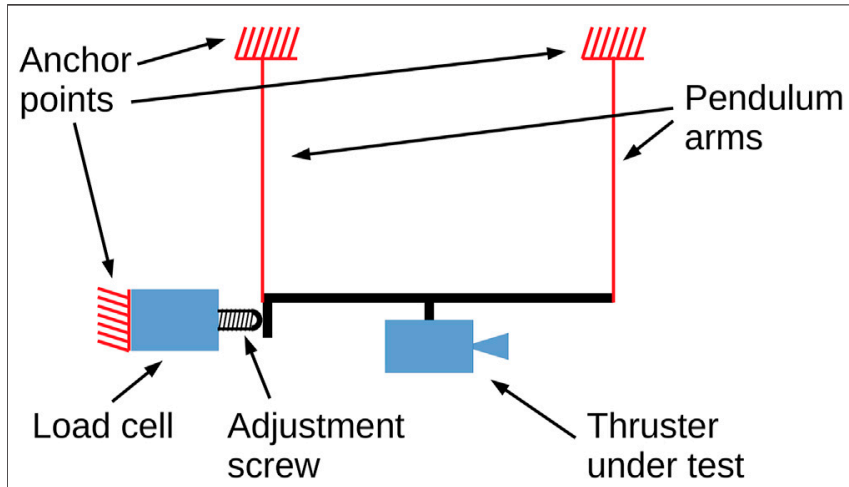

FIGURE 1 | Diagram of thrust balance configured for measurements using the load cell. Only two out of the four pendulum arms are shown. The adjustment screw is firmly attached to the load cell, and it is extended until contact is made with the pendulum frame. It is then extended a second time until the indication on the load cell shows approximately half of its rated full force.

a comparison between direct and indirect thrust measurements, concluding in this case that the two methods produce very similar results.

\subsection{Load Cell in a Standard Weight Scale Configuration Method}

Load cells are force to electrical signal converters, also known as force transducers or force sensors. They are frequently found in electronic scales measuring weight, and they have a range varying from micrograms to tons. They are commonly used to measure thrust from conventional chemical rockets. They can also be used to measure thrust from microthrusters producing less than a millinewton of thrust. One way to achieve thrust measurement using a load cell is to construct a standard weight scale and place the thruster on it with the nozzle facing up (gravitational force is parallel to the thrust force and has the same sign). Since the thruster exhaust faces up, the scale will record an increase in the measured value when the thruster is switched on, which is equal to the thrust. Examples of such thrust stand are given by Conde et al. (2020) and Karadag et al. (2018). The disadvantage of this method is that the measurement system must be able to measure the weight of the thruster $\mathrm{F}_{\mathrm{W}}$, which is typically many orders of magnitude larger than the thrust $\mathrm{F}_{\mathrm{T}}$. The selected load cell must be capable of supporting and measuring $\mathrm{F}_{\mathrm{W}}$, which results in decreased resolution when measuring thrust in the submillinewton range.

\subsection{Load Cell on a Pendulum Method}

A strain gauge or a load cell can also be used to measure the thrust directly in a pendulum stand. Examples of such application have been published for torsional pendulums (Frieler and Groll, 2018) and inverted pendulums (Stephen et al., 2001). The present study describes the direct thrust measurement taken by using a load cell in a conventional hanging pendulum, where the thruster is placed in the hanging pendulum thrust stand and a load cell is placed between the pendulum and a rigid point, along the axis of expected 


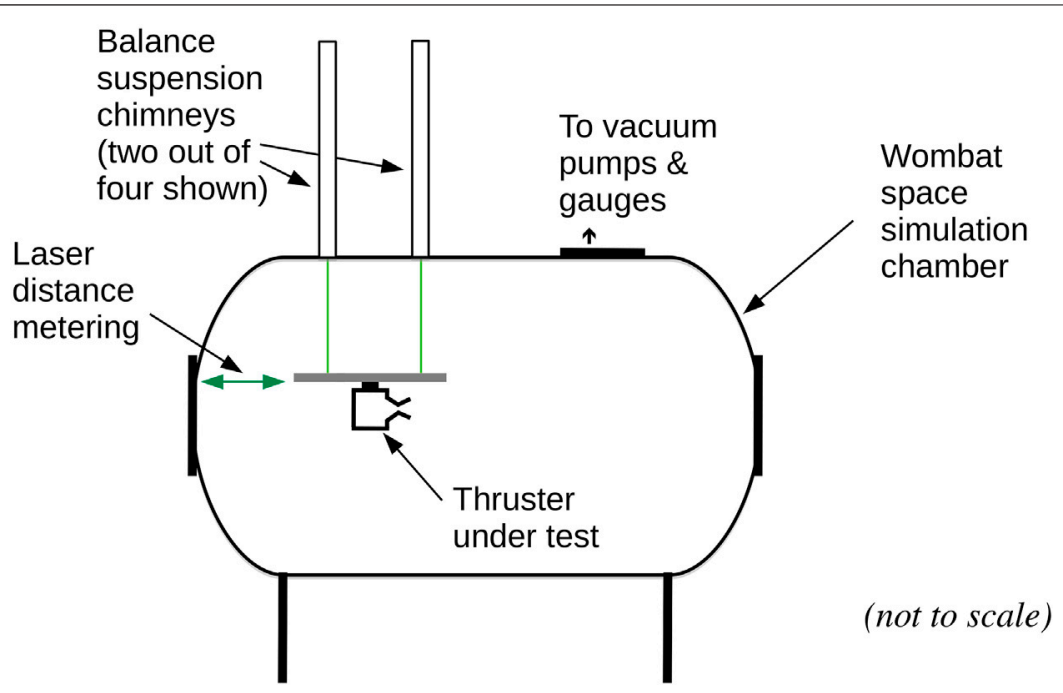

FIGURE 2 | Diagram of the laser distance metering (interferometer) thrust balance in the Wombat space simulation chamber. The cold gas thruster is shown suspended on the balance.

pendulum movement when the thrust force is applied. Measuring the thrust directly instead of measuring the displacement has the advantage that the sensitivity of the metering system is not as strongly dependent on the mass of the thruster that is suspended on the hanging pendulum. This is due to the fact that the movement of the pendulum when configured with the load cell is much less than when it is configured with the laser system. When measuring displacement, the deflection angle $\theta$ (and therefore the displacement) caused by a thrust $\mathrm{F}_{\mathrm{T}}$ from a pendulum with a combined suspended mass $m$ causing a gravity force $F_{G}=m g_{0}$ is given by the formula $\sin \theta=F_{T} / F_{G}=F_{T} / m g_{0}$ which implies smaller angles for increased pendulum suspended mass. In the case of the direct thrust measurement performed using the load cell, $\theta \approx 0$, removing the link of the thrust measurement to the suspended mass.

The hanging pendulum with the load cell configuration can be seen diagrammatically in Figure 1. The load cell is equipped with an adjustment screw which is rotated until contact is made with the pendulum. The pendulum is pushed away from its rest position, and it applies a force to the load cell. The screw is then further adjusted until the measured force is about half of the maximum force allowed on the load cell, allowing enough headroom for the expected thrust. This allows the use of a very sensitive load cell with a low maximum force, which is more suitable for submillinewton measurements. In the present study, thrust is generated and controlled using a well-studied cold gas thruster using argon as the propellant (Ho et al., 2017).

\section{DESCRIPTION OF EXPERIMENT}

\subsection{Wombat Space Simulation Chamber With Cold Gas Thruster}

The thrust measurements were conducted in the Wombat space simulation chamber (Charles et al., 2016; Charles et al., 2013) shown in Figure 2, comprising a $1 \mathrm{~m}$ diameter, $2 \mathrm{~m}$ long vacuum chamber fitted with a dry vacuum pumping system (scroll, turbomolecular, and cryogenic pumps) along with a suite of access and viewing ports for space qualification testing. The vessel is pumped down to a base pressure of about $10^{-6}$ using the $70 \mathrm{~m}^{3} / \mathrm{h}$ scroll and 1,800 l/s turbomolecular pumps but not the cryogenic pump. Wombat is routinely used for the experimental testing of various types of thrusters and their propellant and electric subsystems (Tsifakis et al., 2020) and is equipped with a thrust balance validated in-house (Charles et al., 2013; Charles et al., 2016) and in a number of laboratories (Pottinger et al., 2011; Takahashi et al., 2011).

An electrically grounded chassis can be suspended from the top of 4 'chimneys' and either be made to act as a thrust balance or be locked onto the vacuum vessel. The thrust balance consists of a four-arm pendulum suspended frame with the thruster being mounted on the frame. In operation, the thruster produces a displacement from the rest position, which is proportional to the thrust force, which can be measured by an ILD7100 laser triangulation displacement sensor with a resolution of $0.1 \mu \mathrm{m}$ that is installed on the chamber pointing to a target on the pendulum. The balance has been designed with a built-in calibration system comprising a set of accurately measured weights which are placed on the balance via a pulley and controlled by a stepper motor. This calibration system is used in each measurement to confirm the conversion constant from displacement to force. The calibration process involves installing the thruster to be measured on the balance and then placing the weights on the balance by operating the stepper motor and recording the balance reaction. From this, a calibration factor is produced, which takes into account the mass of the thruster. A set of vacuum feedthrough connections on the vacuum chamber was used to enable electrical connections and thruster control signal transfer from equipment that is located outside the chamber. A 


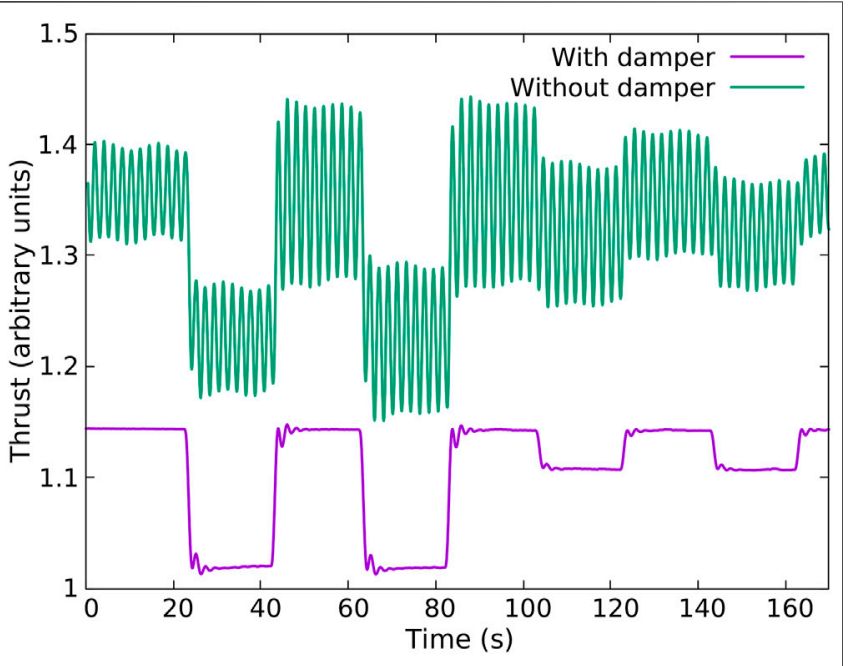

FIGURE 3 | Effect of the magnetic damper when used in the laser interferometer pendulum. The top trace (purple) was recorded without the damper, and the bottom trace (green) was recorded with the damper configuration. In both cases, the cold gas thruster on the stand was setup to do two $20 \mathrm{~s}$ burns at 500 SCCM, followed by two $20 \mathrm{~s}$ burns at 200 SCCM. In both cases, the sampling is set to $312.5 \mathrm{~Hz}$, with a moving average depth of 256

diagram of the Wombat thrust balance showing a thruster on the balance frame and the positioning of the laser distance metering system is seen in Figure 2.

The argon cold gas thruster used for the tests consists of a $4 \mathrm{~mm}$ inner diameter ceramic tube which is fed with a user selectable argon flow rate. The argon is stored in a pressure cylinder located outside the vacuum chamber, and the flow is controlled by using an Alicat Scientific mass flow controller. The mass flow rates used in this experiment range from 25 to 200 SCCM and result in choked flow conditions, as described in Ho et al. (2017) and Tsifakis et al. (2020). A calibration measurement is done at the beginning and end of each measurement campaign to ensure that the correct translation is performed from displacement to thrust, taking into account the exact mass of the thruster as installed for the measurement.

When a constant force is applied to the frame by the thruster, the pendulum moves from its rest position and starts oscillating with a period of about $1 \mathrm{~s}$, which can be damped over a few seconds using a magnet attached to the balance moving in a copper tube. The typical effect of the magnetic damper can be seen in Figure 3, where the cold gas thruster is setup to perform two $20 \mathrm{~S}$ burns at $500 \mathrm{SCCM}$, followed by two burns at 200 SCCM. The top trace (green) shows the interferometer output with the damper removed from the balance, exhibiting a constant oscillation at the pendulum's resonant frequency, which is about $1 \mathrm{~Hz}$. The bottom trace (purple) shows the same thruster sequence with the damper installed. In this case, the oscillation is mostly dampened quickly with a damping factor $\zeta<1$ (underdamping), resulting in a clear trace. There was no change observed to the zero drift as a result of the addition of the damper.

\subsection{Load Cell Setup in Wombat}

To evaluate the direct thrust measurement performance of a load cell, the Wombat thrust balance was modified as follows. A load cell was removed from a diamond pocket scale, which is a lowcost jeweler's scale, purchased online. There were no identifying marks on the load cell, making it impossible to get further information from the manufacturer. However, an assessment of the range and resolution could be made from the advertised properties of the scale. The scale had an advertised maximum allowed weight of $20 \mathrm{~g}$ and a resolution of $1 \mu \mathrm{g}$. The force $\mathrm{F}_{W}$ resulting from the mass $m$ placed on the scale due to gravity could be calculated by using the formula $F_{w}=m g_{0}$, where $g_{0}$ is the standard gravity. This resulted in a maximum force of $\sim 196.2 \mathrm{mN}$ and a resolution of $\sim 9.81 \mu \mathrm{N}$. These operating parameters were compatible with thrust measurement in the submillinewton range. The load cell was a Wheatstone bridge, which was provided with an appropriate excitation voltage and when a force was applied on it, became unbalanced and produced a small voltage which was proportional to the applied force. This load cell did not have any temperature compensation mechanism, which was confirmed by blowing hot air at it with no load, resulting in dramatic changes in the readout. However, this is not a significant drawback in a cold gas thruster evaluation as the load cell will be in vacuum during the measurements, protected from room temperature changes. An Avia Semiconductor HX-711 load cell amplifier/digitizer was used to provide the excitation voltage to the load cell and digitize the resulting force-induced voltage. The digitizer has an advertised 24 bit resolution, but it was discovered in practice that the effective resolution is less than that, with the lower few bits being noise. Even if the effective resolution is 16 bit and assuming the maximum expected thrust force occupies the complete voltage measurement range of the digitizer, the obtainable theoretical resolution is $\sim 3 \mu N$, which is acceptable. The noise amplitude measured with the load cell to the ADC was found to be around $80 \mu \mathrm{N}$ peak-to-peak, which is a factor of four worse than the noise present on the interferometer (around $20 \mu \mathrm{N}$ ) during the measurement campaign.

The amplifier/digitizer was installed inside the Wombat vacuum chamber, very close to the load cell, in order to minimize electrical noise pickup. Although a cold gas thruster does not produce any direct electrical interference, in a lab environment, there are many sources of conducted and radiated noise including large electric motors, switched mode power supplies, and apparatus using radio frequency (RF). A photo of the load cell installation can be seen in Figure 4, where the load cell can be seen (center of photo) rigidly attached to the chamber via an L-bracket on its right side. On the left side of the load cell, the screw that allows precise contact with the pendulum frame is seen. The purpose of the screw is to allow for a high level of control over the preloading of the load cell by pushing against the pendulum. In the photo, the amplifier/digitizer can also be seen on the right side. The digital signals are routed outside the chamber via appropriate feedthrough connectors and are read by using an external microcontroller. The microcontroller is programmed to read the load cell values at a constant rate limited to 10 samples per second by the digitizer and to make them available via a serial port to a computer. The existing 


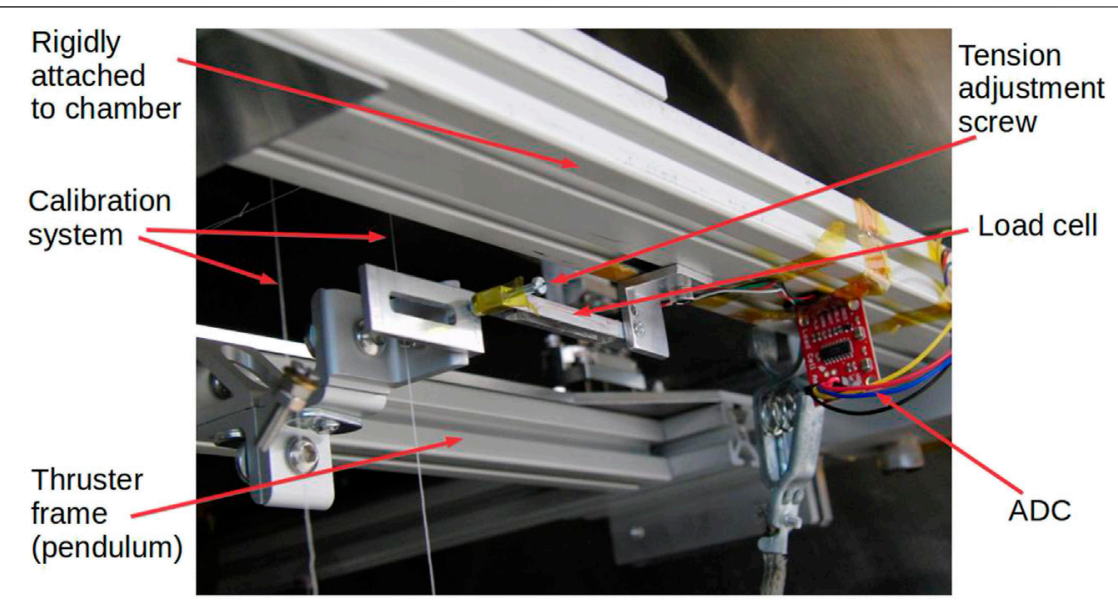

FIGURE 4 | Photo of the load cell installed on the thrust balance. In this photo, the load cell can be seen in the center. The right side of the load cell is rigidly connected to the chamber via an L-bracket, and the left end touches the hanging pendulum via an adjustable screw. The load cell amplifier/digitizer red printed circuit board can be seen in the middle right of the photo. Part of the calibration mechanism can be seen in the middle left of the photo.

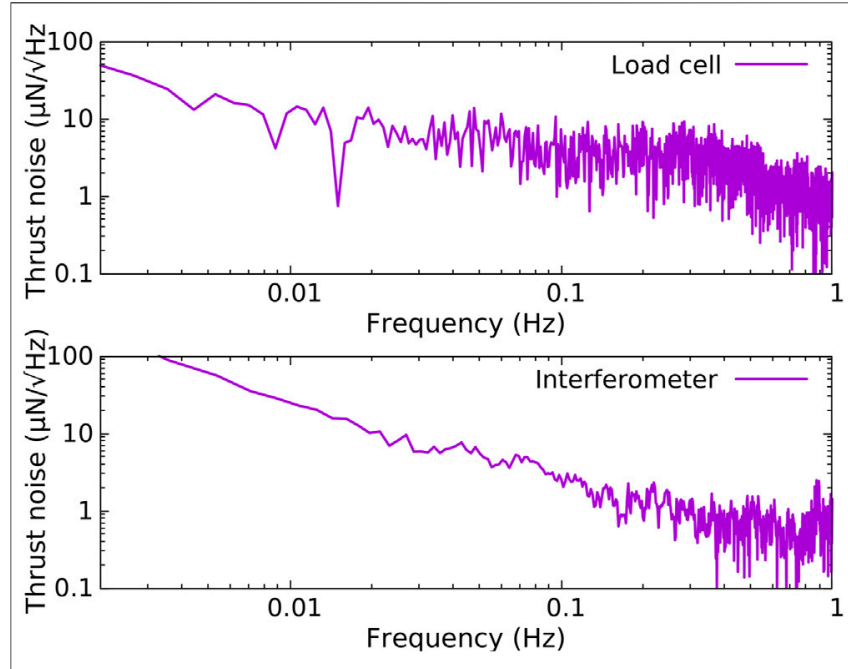

FIGURE 5 | Comparison between the noise spectrum of the load cell (top plot) and that of the laser interferometer (bottom plot). It can be seen that the thrust noise of the load cell is generally higher than that of the interferometer, except for frequencies below $\sim 0.05 \mathrm{~Hz}$, where it is comparable.

calibration system, used for the interferometer calibration and described earlier, was also used to perform the calibration for the load cell.

\section{EXPERIMENTAL RESULTS AND COMPARATIVE ANALYSIS}

Before beginning the comparison of the two systems (load cell and laser interferometer) with an operating thruster, measurements of noise were conducted with the thruster installed but with no gas flowing. In both cases, a $\sim 10 \mathrm{~min}$
TABLE 1 | Resulting thrust from argon flow rates used in this experiment.

\begin{tabular}{lcccc}
\hline $\begin{array}{l}\text { Argon } \\
\text { flow rate (SCCM) }\end{array}$ & $\mathbf{2 5}$ & $\mathbf{5 0}$ & $\mathbf{1 0 0}$ & $\mathbf{2 0 0}$ \\
\hline $\begin{array}{l}\text { Calculated thrust }(\mu N) \\
\text { Interferometer thrust }(\mu N)\end{array}$ & 210 & 415 & 836 & 1,670 \\
Load cell thrust $(\mu N)$ & $210 \pm 50$ & $402 \pm 50$ & $741 \pm 50$ & $1388 \pm 50$
\end{tabular}

The thrust is obtained by repeated measurements for each flow rate, and the error is estimated based on the calibration, noise, and repeatability of each measurement within the same campaign.

period of noise was recorded, calibrated to thrust via the built-in calibration mechanism, and the resulting data were put through a discrete Fourier transformation to obtain the thrust noise spectrum shown in Figure 5. In this figure, the load cell spectrum (top trace) appears to be noisier than the laser interferometer-obtained spectrum (bottom trace). The natural frequency of the pendulum is captured by using the laser interferometer; however, the magnetic damper was installed during this measurement, resulting in a peak at $\sim 0.9 \mathrm{~Hz}$ that is relatively small. The natural frequency of the pendulum is not seen in the load cell spectrum as expected due to the fact that the pendulum is pressed against the load cell and is not allowed to oscillate at such a low frequency.

A $\sim 3 h(\sim 10000 s)$ thrust measuring experiment was then conducted in both systems. Each recording consisted of a calibration run (three weights of $1.17 \mu \mathrm{N}$ on and off the balance) at the beginning and at the end. Between the calibration runs, the system was configured to automatically operate the cold gas argon thruster described earlier with a pattern of 0 SCCM, 25 SCCM, 50 SCCM, 100 SCCM, 200 SCCM, and back to 0 SCCM, each step with a duration of $30 \mathrm{~S}$. The resulting thrust for each flow rate is presented in Table 1. In this table, the thrust shown is obtained by multiple measurements at each flow rate and is measured by using the interferometer and the load cell. The theoretical thrust 


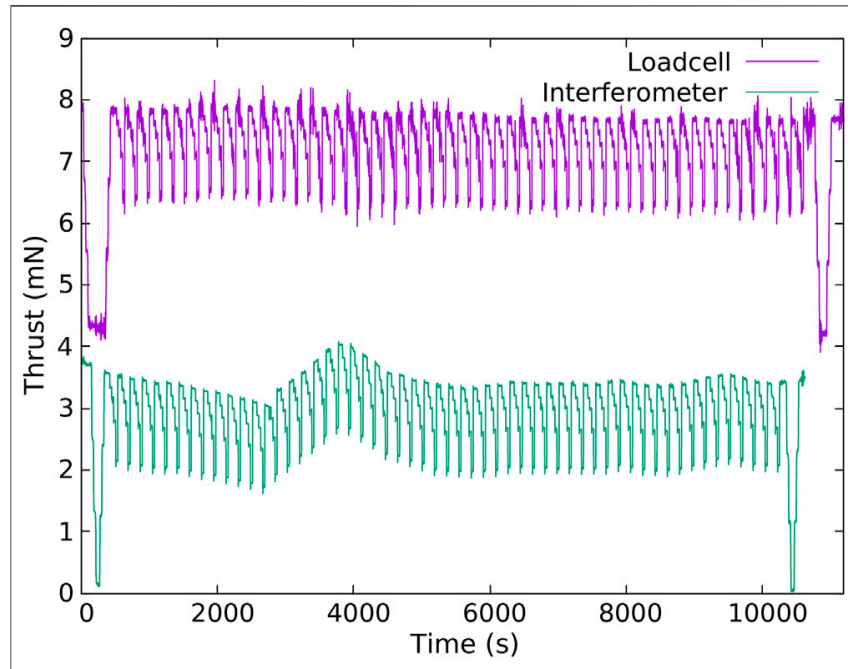

FIGURE 6 | Zero drift comparison between the load cell and laser interferometer thrust measurements. In this figure a $\sim 3 h$ thruster run is shown, using the laser interferometer (bottom trace, green) and the load cell (top trace, purple). Each measurement begins and ends with a calibration run, consisting of three $1.17 \mathrm{mN}$ weights. In both cases, the thruster performs a 0-25-50-100-200-0 SCCM cycle for approximately $3 \mathrm{~h}$. It can be seen that the zero drift of the load cell measurement is much less than that of the laser interferometer. The depicted behavior is typical of the observed behavior of both systems

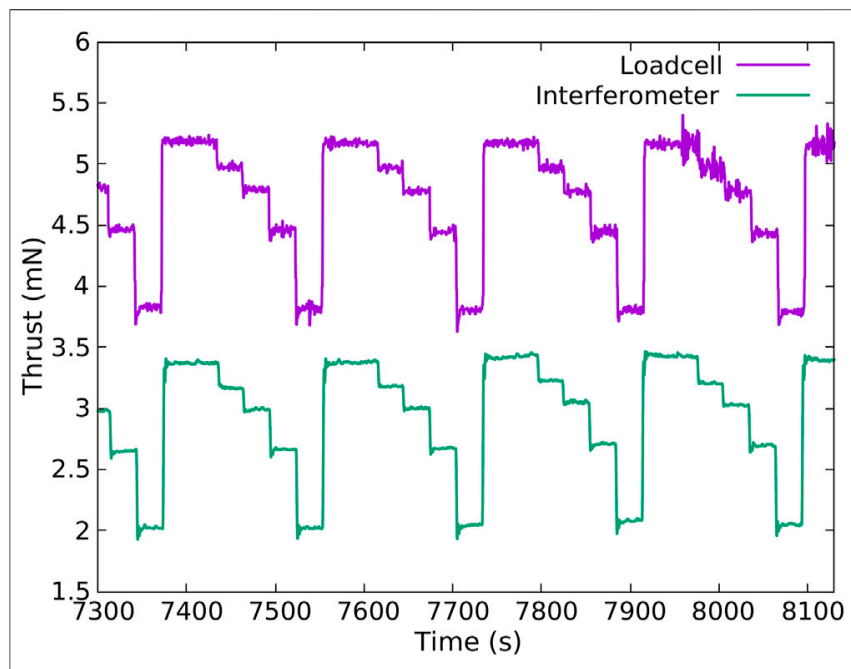

FIGURE 7 | Comparison between the load cell and laser interferometer thrust measurements. This figure focuses on the data shown in Figure $\mathbf{6}$, showing four cycles of the 0-25-50-100-200-0 SCCM cold gas argon thruster sequence. It can be seen that the noise in the load cell measurement (top trace, purple) is higher roughly by a factor of two than that in the laser system (bottom trace, green). It can also be seen that the load cell can suddenly suffer increased noise (as seen in the plot around 8,000 s); however, this has little effect in a repeated measurement.

is also presented, calculated by using the method described in Tsifakis et al. (2020). The agreement between the two measurement methods is very good, and the overestimation of

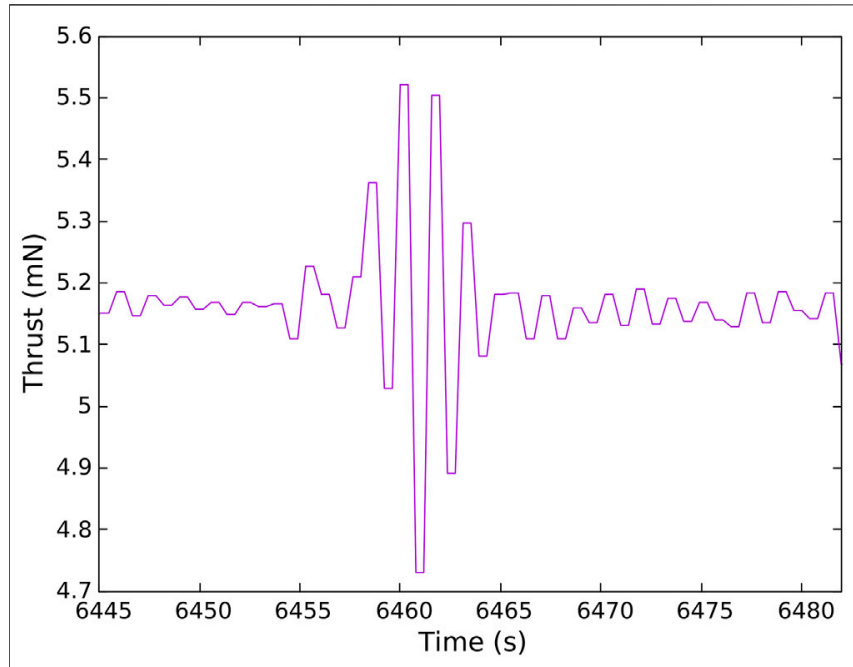

FIGURE 8 | Focus on a 'microslip.' This figure shows a typical sudden noise observation which happens at a time when there is no change in the forces applied to the load cell from the thruster. It is believed to be a 'microslip,' occurring when the forces at the load cell-pendulum contact boundary are unbalanced and the net force suddenly overcomes the friction at the contact point between the load cell and the pendulum. This is a process that resembles an earthquake induced along a fault line.

the theoretical calculation can be attributed to the boundary layer friction force described in detail in Ho, et al. (2017), which is not taken into account in the calculation.

The $30 \mathrm{~s}$ step duration was selected to allow the pendulum to settle for an accurate measurement of the thrust. This sequence was repeated for approximately $30 \mathrm{~h}$, and the resulting data are shown in Figure 6. In this figure, the interferometer data (top green trace) appear to be drifting slowly during the measurement. This effect, known as zero drift, is likely caused by temperature changes throughout the measurement. The load cell data (bottom purple trace) exhibit limited zero drift, despite not being temperature compensated. This behavior is typical of the observations derived from using both systems for an extended period of time in the laboratory.

Figure 7 focuses on a few periods of the repeated measurement cycle. As expected, the laser interferometer (top green trace) exhibits less noise compared to the load cell (bottom purple trace) by roughly a factor of two. The noise behavior is consistent in the laser interferometer and in part can be reliably traced to changes in the vicinity of the chamber, such as people walking around the laboratory (floor deformation) or the laboratory door being opened. These actions also have a measurable effect on the load cell measurement; however, there are additional noise events which cannot be linked to known external factors and appear at seemingly random points throughout the recording. Such an event can be seen in Figure 7, around $t=8000 \mathrm{~s}$. For a long recording, the appearance of these events does not have a detrimental effect on the accuracy of the measurement as the affected parts of the data can be easily identified and excluded from the rest of the measurement.

Figure 8 shows a single episode of this sudden noise. This example is typical of this type of noise and happens at a time 
when there is no change in the applied force on the balance. The working hypothesis on the origin of this noise is that it is the result of a 'micro-slip' at the contact boundary between the load cell adjustment screw and the pendulum scale (Figures 1, 4). This process resembles that of an earthquake produced when the two sides of a geological fault suddenly slip laterally, commonly called a 'strike-slip'.

The experiment presented in this paper involves a cold gas thruster. In order to use this technique with other types of thrusters, the thrust balance designer needs to consider the thermal and electromagnetic compatibility of the thruster and the load cell. In particular, thrusters such as plasma thrusters may produce excessive heat during operation, which will affect the measurements of a non-temperature-compensated load cell. Any RF plasma thruster or other pulsed thrusters can cause electromagnetic interference (EMI) which, if picked up by the load cell amplifier, can result in increased measurement noise. Plasma thrusters tested in a vacuum chamber can create a low-density plasma which may result in corrosion of the load cell and associated electronics. In all these cases, the thrust balance designer will have to supply appropriate shielding to minimize the heat and electrical noise pickup and the plasma-induced damage. In part, better load cell protection can be achieved by the installation of the load cell and associated electronics as far away as practical from the thruster being tested.

\section{CONCLUSION}

This paper has presented an alternative thrust measurement system for a hanging pendulum thrust stand based on a

\section{REFERENCES}

Asakawa, J., Nishii, K., Nakagawa, Y., Koizumi, H., and Komurasaki, K. (2020). Direct Measurement of 1-mN-Class Thrust and 100-S-Class Specific Impulse for a CubeSat Propulsion System. Rev. Sci. Instr. 91, 035116. doi:10.1063/1. 5121411

Bonin, B., Roth, N., Armitagee, S., Newman, J., Zee, B. R,, Robert, E., et al. (2015). "CanX-4 and CanX-5 Precision Formation Flight: Mission Accomplished!," in Proceedings of the 29th Annual AIAA/USU Conference on Small Satellites, Logan, Utah, July 31 2015. doi:10.1039/c3tc30232f

California Polytechnic State University (2014). Cubesat Design Specification Rev. 13. Available at: http://www.cubesat.org/s/cds $\left\{\_\right\}$rev13\{\_\}final2.pdf.

Charles, C., Boswell, R. W., and Bish, A. (2013). Variable Frequency Matching to a Radiofrequency Source Immersed in Vacuum. J. Phys. D: Appl. Phys. 46, 365203. doi:10.1088/0022-3727/46/36/365203

Charles, C., Boswell, R. W., Bish, A., Khayms, V., and Scholz, E. F. (2016). Direct Measurement of Axial Momentum Imparted by an Electrothermal Radiofrequency Plasma Micro-Thruster. Front. Phys. 4, 19. doi:10.3389/fphy. 2016.00019

Chavers, D. G., and Chang-Díaz, F. R. (2002). Momentum Flux Measuring Instrument for Neutral and Charged Particle Flows. Rev. Sci. Instr. 73, 3500-3507. doi:10.1063/1.1505107

Conde, L., Lahoz, M. D., Grabulosa, J., Hernández, R., González, J., Delgado, M., et al. (2020). Thrust Stand Based on a Single Point Load Cell for Impulse Measurements from Plasma Thrusters. Rev. Sci. Instr. 91, 023308. doi:10.1063/ 1.5127189

Frieler, T., and Groll, R. (2018). A Torsional Sub-milli-Newton Thrust Balance Based on a Spring Leaf Strain Gauge Sensor. Rev. Scientific Instr., 89, 075101. doi:10.1063/1.4996419 low-cost load cell. Its performance is directly compared to the more typical system of measuring the induced displacement of the pendulum by the thruster using a laser interferometer. While the laser system still has the advantage of lower noise by roughly a factor of two, the load cell was found to exhibit less zero drift despite not having temperature compensation. It also has a considerably lower cost than the interferometer, making it a viable alternative, especially for thrust measurements in the range of hundreds of micronewtons. Another advantage is that the load cell-based stand sensitivity is not as dependent on the suspended mass (weight), unlike the displacement measuring system. Alternative load cells can be utilized to modify the measurement range, if that is required. The load cell system was found to suffer from seemingly random breakouts of noise; however, this does not affect the result of a repeated measurement.

\section{DATA AVAILABILITY STATEMENT}

The original contributions presented in the study are included in the article/Supplementary Material; further inquiries can be directed to the corresponding author.

\section{AUTHOR CONTRIBUTIONS}

The work presented in this paper is DT's student work and has been supervised by CC and RB. All authors contributed to the article and approved the submitted version.

Haag, T. W. (1991). Thrust Stand for High-power Electric Propulsion Devices. Rev. Sci. Instr. 62, 1186-1191. doi:10.1063/1.1141998

Ho, T. S., Charles, C., Teck, S. H., Roderick, W., and Boswell (2017). A Comprehensive Cold Gas Performance Study of the Pocket Rocket Radiofrequency Electrothermal Microthruster. Front. Phys. doi:10.3389/fphy. 2016.00055

Karadag, B., Cho, S., and Funaki, I. (2018). Note: Precision Balance for SubmiliNewton Resolution Direct Thrust Measurement. Rev. Sci. Instr. 89, 086108. doi:10.1063/1.5045855

Kokal, U., and Celik, M. (2017). "Development of a Mili-Newton Level Thrust Stand for Thrust Measurements of Electric Propulsion Systems," in Proceedings of 8th International Conference on Recent Advances in Space Technologies, Istanbul, Turkey, June 2017. doi:10.1109/RAST.2017. 8002970

Kramer, A., Bangert, P., and Schilling, K. (2020). UWE-4: First Electric Propulsion on a $1 \mathrm{U}$ CubeSat-In-Orbit Experiments and Characterization. Aerospace 7, 98. doi:10.3390/aerospace7070098

Kulu, E. (2019). Nanosat Database. Available at: https://www.nanosats.eu. (Accessed April 4, 2021).

Little, B., and Jugroot, M. (2019). Development of a Microthrust Balance and Ion Beam Measurement System: Characterizing a Dual-Mode Thruster for Spacecraft. Vacuum 164, 367-380. doi:10.1016/j.vacuum.2019.01.031

Moeller, T., and Polzin, K. A. (2010). Thrust Stand for Vertically Oriented Electric Propulsion Performance Evaluation. Rev. Sci. Instr. 81, 115108. doi:10.1063/1. 3502463

Polk, J. E., Pancotti, A., Haag, T., King, S., Walker, M., Blakely, J., et al. (2017). Recommended Practice for Thrust Measurement in Electric Propulsion Testing. J. Propuls. Pow. 33, 539-555. doi:10.2514/1.B35564

Pottinger, S., Lappas, V., Charles, C., and Boswell, R. (2011). Performance Characterization of a Helicon Double Layer Thruster Using Direct Thrust 
Measurements. J. Phys. D: Appl. Phys. 44, 235201. doi:10.1088/0022-3727/44/ $23 / 235201$

Soni, J., and Roy, S. (2013). Design and Characterization of a Nano-Newton Resolution Thrust Stand. Rev. Sci. Instr. 84, 095103. doi:10.1063/1.4819252

Stephen, R. J., Rajanna, K., Dhar, V., Kumar, K. G. K., and Nagabushanam, S. (2001). Strain Gauge Based Thrust Measurement System for a Stationary Plasma Thruster. Meas. Sci. Technol. 12, 1568-1575. doi:10.1088/0957-0233/ $12 / 9 / 324$

Takahashi, K., Lafleur, T., Charles, C., Alexander, P., Boswell, R. W., Perren, M., et al. (2011). Direct Thrust Measurement of a Permanent Magnet Helicon Double Layer Thruster. Appl. Phys. Lett. 98, 141503. doi:10.1063/1.3577608

Takahashi, K., Komuro, A., and Ando, A. (2015). Measurement of Plasma Momentum Exerted on Target by a Small Helicon Plasma Thruster and Comparison with Direct Thrust Measurement. Rev. Sci. Instr. 86, 023505. doi:10.1063/1.4907797

Tang, H., Shi, C., Zhang, X. a., Zhang, Z., and Cheng, J., (2011). Pulsed Thrust Measurements Using Electromagnetic Calibration Techniques. Rev. Sci. Instr. 82 (3), 035118. doi:10.1063/1.3567803

Tsifakis, D., Charles, C., and Boswell, R. (2020). An Inductively-Coupled Plasma Electrothermal Radiofrequency Thruster. Front. Phys. 8, 34. doi:10.3389/fphy. 2020.00034

West, M. D., Charles, C., and Boswell, R. W. (2009). A High Sensitivity Momentum Flux Measuring Instrument for Plasma Thruster Exhausts and Diffusive Plasmas. Rev. Sci. Instr. 80, 053509. doi:10.1063/1.3142477
Wong, A. R., Toftul, A., Polzin, K. A., and Pearson, J. B. (2012). Non-contact Thrust Stand Calibration Method for Repetitively Pulsed Electric Thrusters. Rev. Sci. Instr. 83, 025103. doi:10.1063/1.3680557

Zhang, Z., Ren, J., Tang, H., Ling, W. Y. L., York, T. M., and York (2017). An Ablative Pulsed Plasma Thruster with a Segmented Anode. Plasma Sourc. Sci. Technol. 27 (1), 015004. doi:10.1088/1361-6595/aa9e6b

Zhou, W.-J., Hong, Y.-J., and Chang, H. (2013). A microNewton Thrust Stand for Average Thrust Measurement of Pulsed Microthruster. Rev. Sci. Instr. 84, 125115. doi: $10.1063 / 1.4850637$

Ziemer, K. J. (2001). "Performance Measurements Using a Sub-Micronewton Resolution Thrust Stand," in 27th International Electric Propulsion Conference, Pasadena, CA, October 15-19, 2001.

Conflict of Interest: The authors declare that the research was conducted in the absence of any commercial or financial relationships that could be construed as a potential conflict of interest.

Copyright (c) 2021 Tsifakis, Charles and Boswell. This is an open-access article distributed under the terms of the Creative Commons Attribution License (CC BY). The use, distribution or reproduction in other forums is permitted, provided the original author(s) and the copyright owner(s) are credited and that the original publication in this journal is cited, in accordance with accepted academic practice. No use, distribution or reproduction is permitted which does not comply with these terms. 